\title{
Radiofrequency Catheter Ablation of AV Nodal Reentry: The Anterior Approach
}

\author{
JONATHAN J. LANGBERG \\ From the Department of Internal Medicine, Division of Cardiology, The University of Michigan \\ Medical Center, Ann Arbor, Michigan
}

\section{Introduction}

The ability to cure atrioventricular (AV) nodal reentry with radiofrequency catheter ablation with preservation of antegrade conduction represents a significant clinical advance. It also provides an opportunity to better understand the pathophysiology of this fascinating arrhythmia.

Two techniques have been developed for ablation of AV nodal reentry. The first to be described used lesions made anteriorly near the apex of the triangle of Koch. More recently, lesions applied posteriorly, adjacent to the ostium of the coronary sinus, have been used.

The purpose of this article is to describe the technique and electrophysiological effects of the anterior approach. Methods for minimizing the risk of high grade AV block will also be discussed. Results of a prospective comparison of anterior and posterior lesions for treatment of AV nodal reentry will be reviewed. Based on these data, recommendations for the clinical role of the anterior approach will be made.

\section{Technique}

One of the virtues of the anterior approach is its simplicity. The ablation catheter is positioned at the site of the maximal His bundle electrogram and is then withdrawn several millimeters proximally. The effective site is usually, but not invariably, slightly anterior to the location of the maximal His bundle electrogram (Fig. 1). Bipolar electrograms recorded during manipulation of the ablation catheter are shown in Figure 2. Target sites

Address for reprints: Jonathan J. Langberg, M.D., University of Michigan Medical Center, 1500 East Medical Center Drive, B1 F245, Ann Arbor, MI 48109. Fax: (313) 936-7641. are characterized by an atrial:ventricular electrogram amplitude ratio $>1$ and a His bundle electrogram amplitude $<100 \mu \mathrm{V}$.

Radiofrequency energy application at the effective site usually results in the occurrence ofjunctional tachycardial However, this is a nonspecific finding, occurring during $25 \%$ of energy applications at ineffective sites. It is advisable to pace the atrium during junctional tachycardia to allow continuous monitoring of antegrade AV conduction. The endpoint for radiofrequency current delivery should be PR prolongation by about $50 \%$ over baseline. Of course, energy delivery must be stopped immediately if a nonconducted $\mathrm{P}$ wave is observed.

A recent preliminary report from Erlich and co-workers ${ }^{2}$ describes the application of radiofrequency energy anteriorly at the site of earliest retrograde activation during AV nodal reentry tachycardia. It has been our practice to avoid ablation during supraventricular tachycardia because of the difficulty with stabilizing the catheter and the risk of dislodgement during energy delivery with termination of the arrhythmia.

\section{Electrophysiological Effects of Anterior Lesions}

Radiofrequency catheter ablation anteriorly produces distinct electrophysiological effects. The AH interval increases by a mean of $35 \%$ over baseline. Despite this increase in antegrade conduction time, there is no change in the mean AV nodal Wenckebach block cycle length or the antegrade effective refractory period of the AV node. In contrast, retrograde AV nodal function is dramatically attenuated by anterior lesions. Forty-five percent of patients have complete elimination of ventriculoatrial conduction after ablation. The remainder have significant attenuation of retrograde func- 


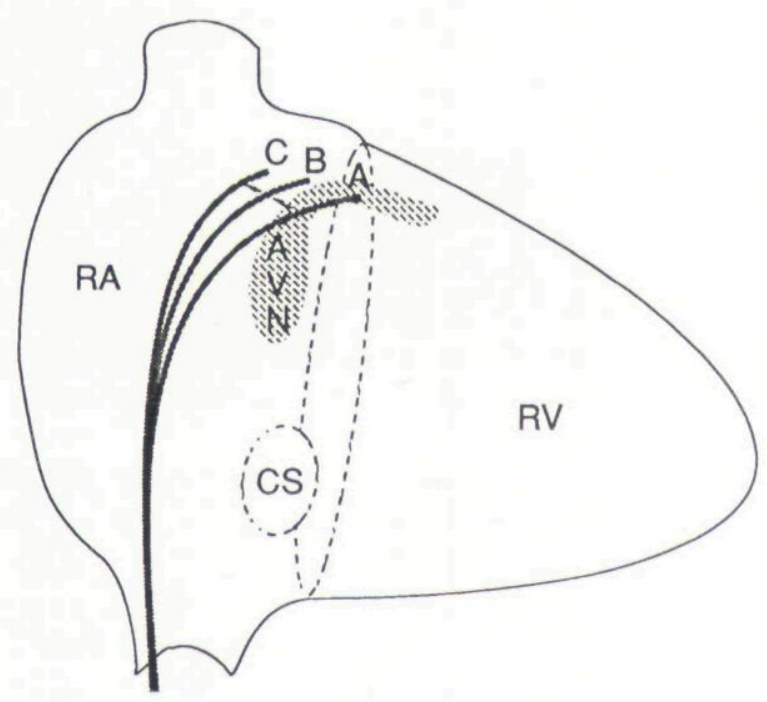

Figure 1. Manipulation of the ablation catheter from the site of the maximal His bundle electrogram (A) to the site used for ablation of the fast AV nodal pathway (C). $A V N=$ atrioventricular node; $C S=$ ostium of the coronary sinus; $R A=$ right atrium; $R V=$ right ventricle. tion, as manifest by an increase in the ventriculoatrial Wenckebach block cycle length and ventriculoatrial conduction time. Although dual AV nodal physiology persists after ablation in a few patients, most have a continuous curve that appears similar to the slow pathway portion of the AV nodal function curve before ablation (Fig. 3).

These observations suggest that anterior lesions have a selective effect on fast AV nodal pathway function rather than damaging the node in a nonspecific fashion.

After successful anterior ablation, a new reentrant paroxysmal supraventricular tachycardia was induced in about $5 \%$ of patients. ${ }^{3}$ This arrhythmia is characterized by a short PR interval and a long RP interval, giving it the appearance of atypical AV nodal reentry (Fig. 4). The new AV nodal reentry was induced exclusively with critically timed ventricular extrastimuli and only during infusion of isoproterenol (Fig. 5). In slightly more than half of the patients studied, the atypical reentry was associated with the emergence of new retrograde dual AV nodal physiology. These patients had the same sequence of retrograde atrial activation before and after ablation with the earli-
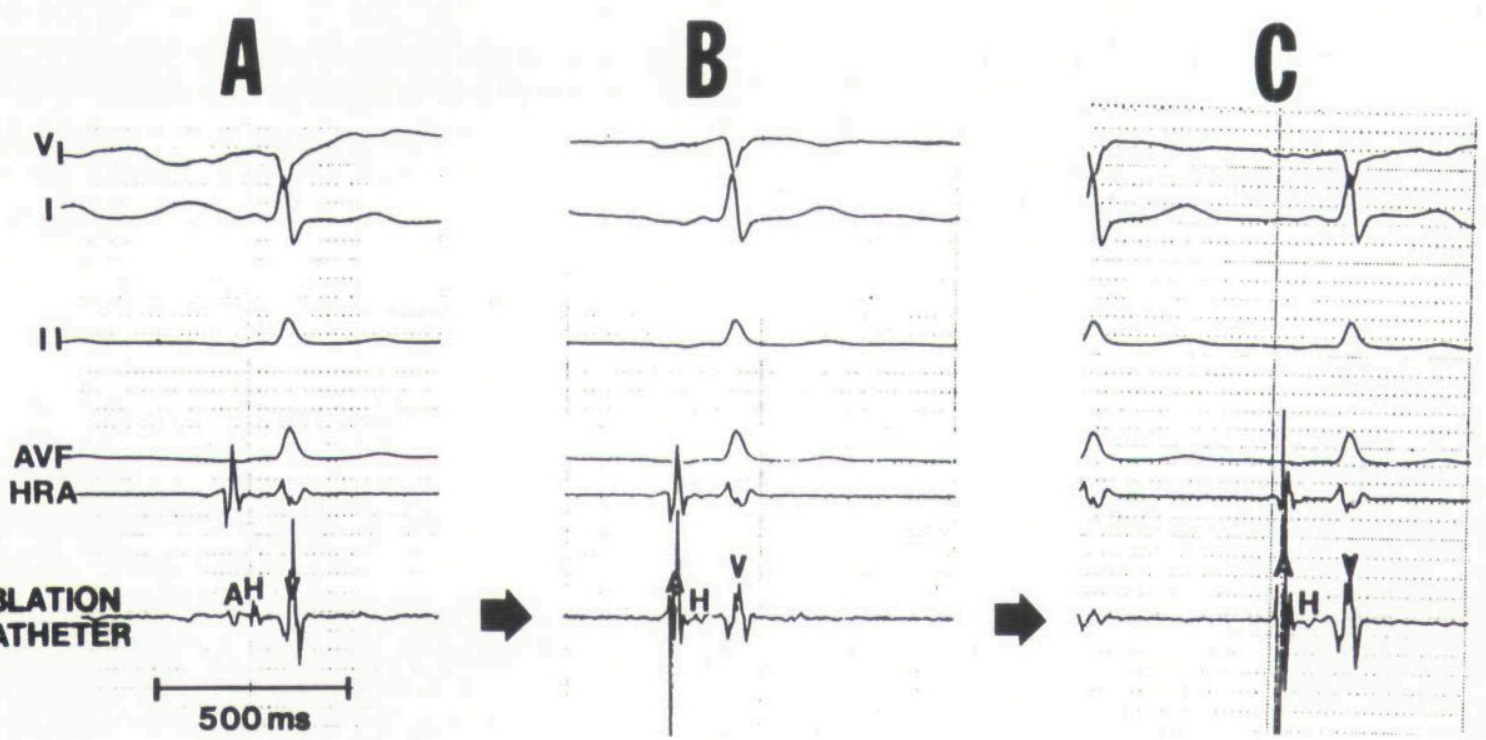

Figure 2. Bipolar endocardial electrograms recorded during manipulation of the ablation catheter at the site of the maximal His bundle electrogram (A). The ventricular electrogram (V) is relatively large and the atrial electrogram $(A)$ is small. As the catheter is withdrawn proximally and anteriorly $(B)$, the His $(H)$ and ventricular electrograms diminish in size and the atrial electrogram grows. At the target site (C), the atrial signal is more than twice the size of the ventricular signal and the His bundle electrogram is barely visible. 


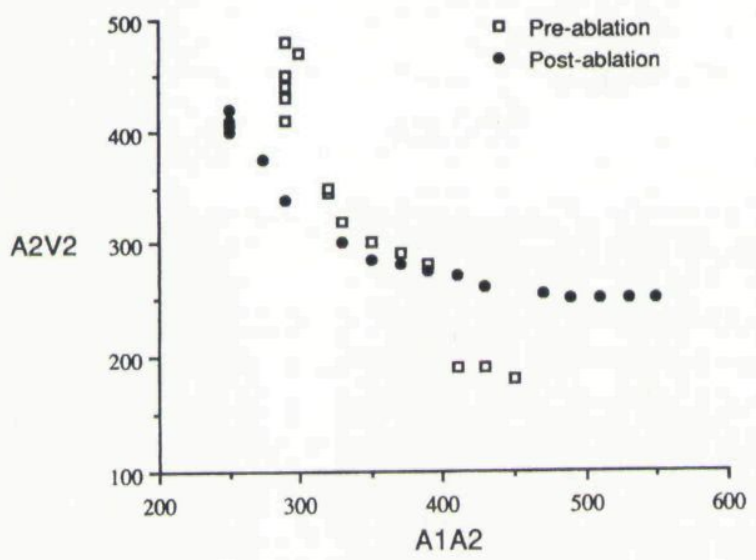

Figure 3. Atrioventricular nodal function curves before and after anterior ablation. The AV nodal function curve before ablation is represented by the open squares and after ablation by the solid circles. Note the disappearance of the fast pathway portion of the curves after ablation. est atrial activation during tachycardia being recorded from the His bundle catheter. In this subgroup of patients, anterior ablation may have attenuated but not completely destroyed fast pathway function. By increasing retrograde fast pathway refractoriness, a premature ventricular stimulus may conduct exclusively up the slow pathway and then down the damaged fast pathway (Fig. $6 \mathrm{~A})$.

Other patients with atypical tachycardia after anterior ablation had a shift in retrograde activation sequence from anterior to posterior during atypical reentry. Most likely these are patients with multiple slow pathways posteriorly. Destruction of fast pathway function with anterior lesions unmasked a second, slower retrograde limb posteriorly that mediated the atypical reentry (Fig. 6B).

These results confirm that the regions mediating fast and slow pathway function are anatomically distinct, usually being separated by 3 to 5 $\mathrm{cm}$.

Only 1 of the 11 patients with atypical reentry induced during follow-up electrophysiology test-
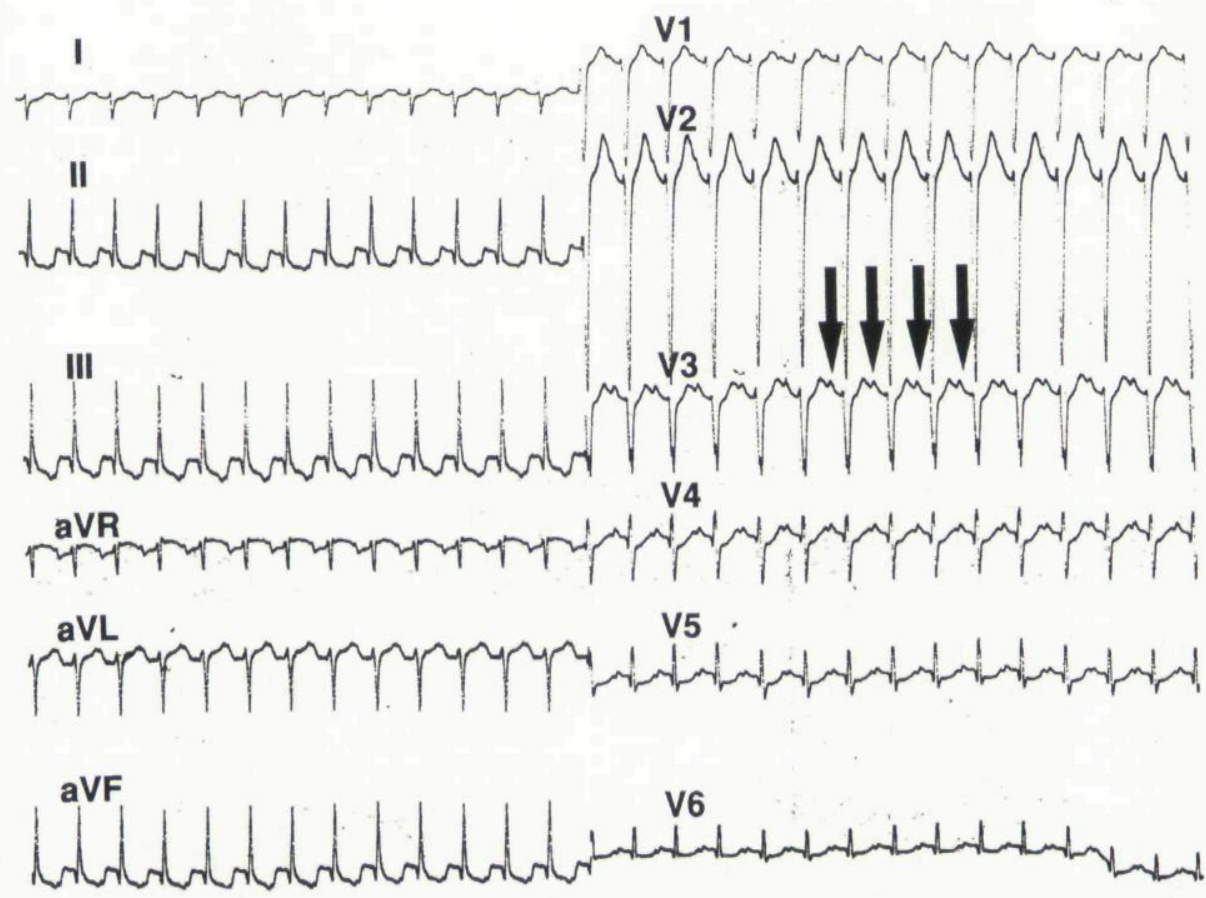

Figure 4. Twelve-lead ECG during atypical AV nodal reentry induced after radiofrequency catheter ablation. Note the short PR interval (arrows) and the superior P wave axis. 


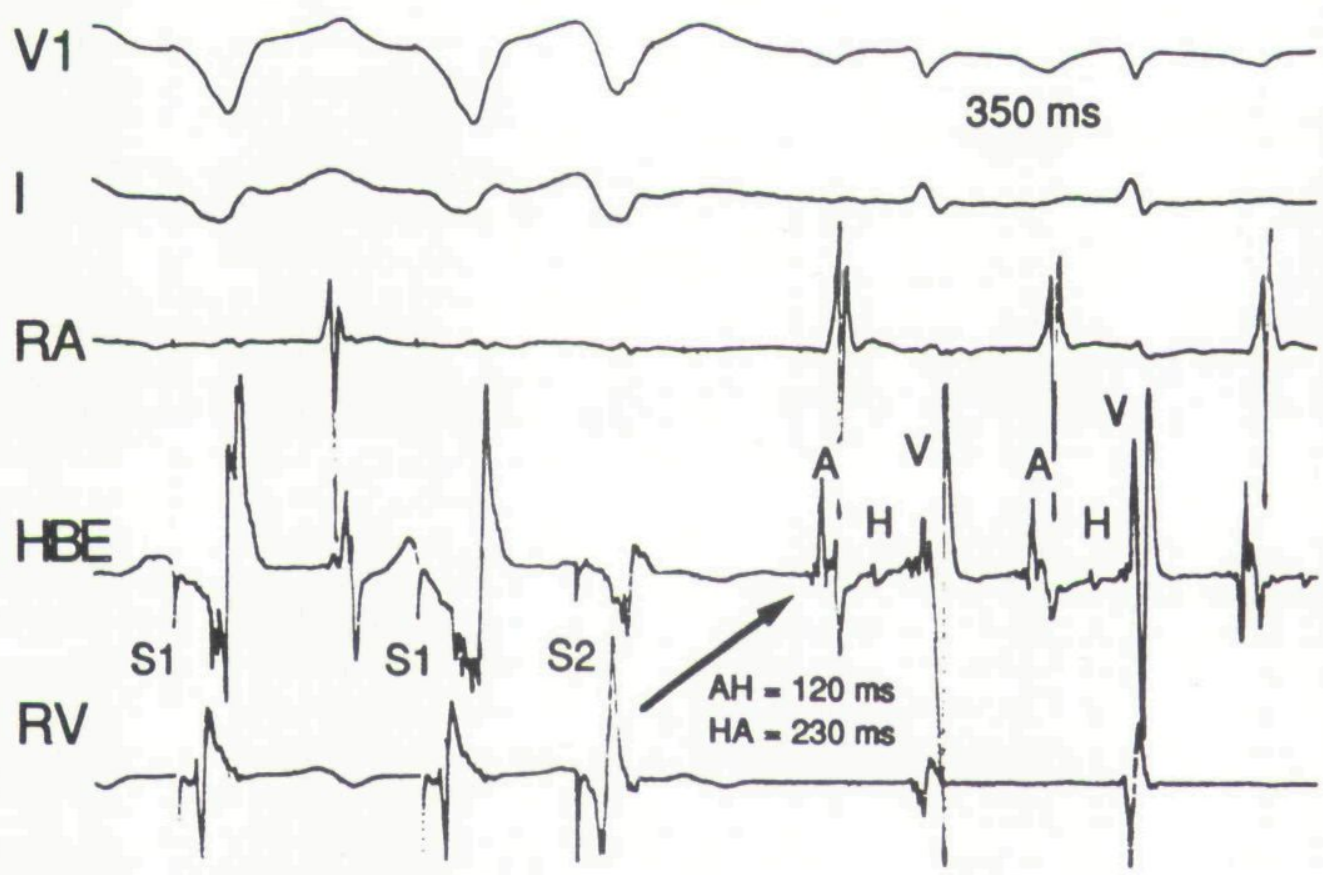

Figure 5. Induction of atypical AV nodal reentry with ventricular extrastimulation. Note the initiation of tachycardia with prolongation of the ventriculoatrial activation time (arrow) resulting in an "up slow-down fast" reentry. $A H=$ atrio-His interval; $H A=$ His-atrial interval; $R A=$ right atrial electrogram; $H B E=$ His bundle electrogram; $R V=$ right ventricular endocardial recording.

ing had a spontaneous arrhythmia. These data suggest that typical AV nodal reentry can be controlled without complete destruction of reentrant pathways. Since induction of atypical AV nodal reentry after anterior ablation appears to be of little clinical consequence, it should not be an indication for drug therapy or additional catheter ablation.

\section{AV Block Complicating Anterior Ablation}

Although the anterior approach is effective, reports from our laboratory and others have suggested that it is associated with a risk of inadvertent complete AV block ranging from $8 \%$ to $21 \%$. ${ }^{1,4}$ Initially, we used a fixed power output at each target site. In an attempt to reduce the risk of high grade AV block, the energy delivery paradigm was then changed such that power output was gradually incremented during each application. The safety and effectiveness of this new approach was compared retrospectively to the original technique. ${ }^{5}$

In patients 1 through 89, a fixed radiofrequency power output of 20 to 30 watts was applied at each target site. If junctional ectopy or a change in the PR interval was seen, then energy delivery was continued until the PR interval increased by $50 \%$ over baseline. If no effect was seen after 20 seconds, the ablation catheter was repositioned and the process repeated.

A different protocol was used in patients 90 through 127. At each target site, power was initially applied at 10 watts for 10 to 15 seconds. If no effects were seen, power was increased by 2 to 4 watts every 10 to 15 seconds up to a maximum of 25 to 30 watts. When junctional ectopy or an increase in the PR interval was seen, no further increments in power were made and the output was continued at this same level for an additional 10 to 15 seconds. 

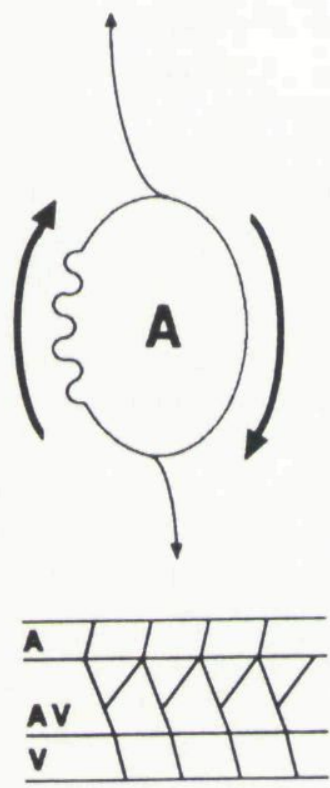
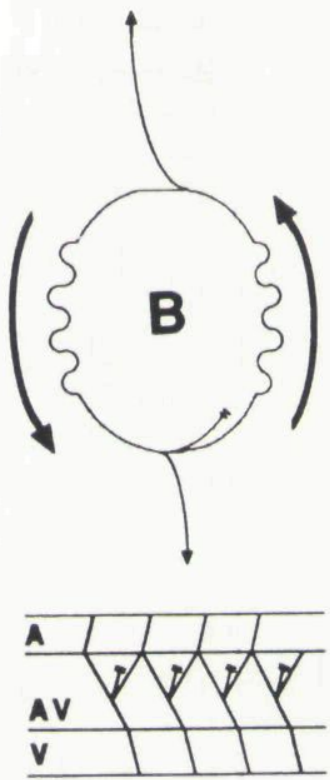

Figure 6. Possible mechanisms of atypical AV nodal reentry after ablation. (A) Ablation induced increase in fast pathway refractoriness might result in selective retrograde fast pathway block and initiation of an "up slow-down fast" reentry. (B) Anterior ablation may destroy the fast pathway, unmasking a second, slower retrograde pathway posteriorly. $A=$ atrium; $A V=$ atrioventricular node; $V=$ ventricle.

The results of the ablation session for the two cohorts are summarized in Table I. A mean of 6.8 \pm 4.9 applications were used in the patients treated with a fixed power output compared with $6.3 \pm 4.4$ applications in those in whom titration was used. Procedure duration, defined as the time from insertion of hemostatic sheaths until completion of the postablation electrophysiology test, was significantly shorter at $61 \pm 46$ minutes in the subjects treated using the incremental approach compared with $88 \pm 37$ minutes in subjects in whom a fixed power output was used $(\mathrm{P}=0.008)$.

Primary success, defined as elimination of inducible AV nodal reentry with preservation of 1 : 1 conduction in sinus rhythm, was achieved in comparable proportions of both cohorts. Inadvertent complete AV block was produced in nine $(10 \%)$ of the 89 patients in whom a fixed power

Table I.

Outcome of Radiofrequency Catheter Ablation of AV Nodal Reentry Tachycardia Using a Fixed Power Output Compared With an Incrementally Increasing Power Output

\begin{tabular}{lccc}
\hline & $\begin{array}{c}\text { Fixed } \\
\text { Power Output }\end{array}$ & $\begin{array}{c}\text { Incremental } \\
\text { Power Output }\end{array}$ & P Value \\
\hline Number of Subjects & 89 & 38 & \\
Number of Radiofrequency Applications & $6.8 \pm 4.9$ & $6.3 \pm 4.4$ & $\mathrm{NS}$ \\
Procedure Duration & $88 \pm 37 \mathrm{~min}$ & $61 \pm 46 \mathrm{~min}$ & 0.008 \\
Primary Success & $74 / 80(92 \%)$ & $37 / 38(96 \%)$ & $\mathrm{NS}$ \\
Complete AV Block & $9 / 89(10 \%)$ & $0 / 38$ & 0.042 \\
\hline
\end{tabular}


output was used. The frequency of this complication did not appear to diminish with increasing experience; two cases occurred in patients 1 through 30 , three in patients 31 through 60 , and four in patients 61 through 89. In contrast, none of the subsequent 38 patients treated using the incremental technique developed complete AV block $(\mathrm{P}=0.042)$.

These data suggest that starting at lower power and gradually incrementing the output during radiofrequency energy application anteriorly reduces the risk of complete AV block without compromising efficacy.

\section{Comparison of the Anterior and Posterior Approaches to AV Nodal Modification}

Several other laboratories have shown that radiofrequency lesions applied posteriorly, near the ostium of the coronary sinus, can effectively eliminate slow pathway function in AV nodal reen- try..$^{4,6,7}$ We compared the effectiveness and safety of the anterior and posterior techniques in a prospective, randomized study. ${ }^{8}$

After confirming the presence of typical AV nodal reentry at the baseline electrophysiology test, patients were randomized to receive lesions anteriorly, using the technique described above, or posteriorly. Posterior target sites were sought with atrial:ventricular electrogram ratios $<1$ and late components in the atrial electrogram that were inscribed after the offset of the septal atrial electrogram and before the His bundle activation.

After ten ineffective energy applications or 1 hour, the position of the ablation catheter was crossed over from anterior to posterior or vice versa.

Of the 50 patients with typical AV nodal reentry tachycardia enrolled in the study, 22 were randomized to receive lesions anteriorly and 28 were initially treated with radiofrequency energy applications applied posteriorly. As seen in Figure 7, primary success was achieved in comparable pro-

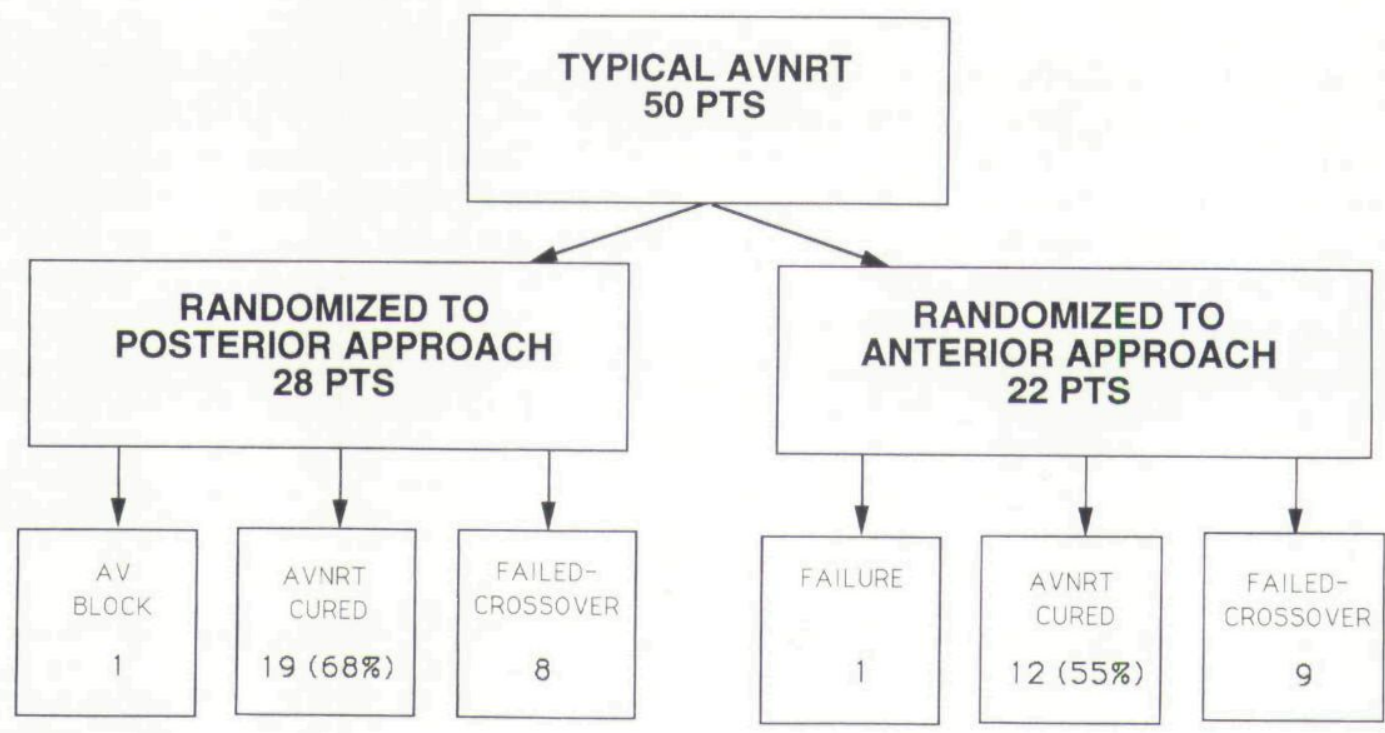

- $6 \pm 3$ RF applications

- 37 \pm 24 minute ablation duration - $22 \pm 10$ minute fluoroscopy exposure
- $6 \pm 4$ RF applications

- $40 \pm 38$ minute ablation duration

- $20 \pm 14$ minute fluoroscopy exposure

Figure 7. Initial outcome of patients randomized to receive lesions anteriorly or posteriorly. Note that the primary success rate was comparable in the two groups, as were the number of energy applications, the procedure durations, and the fluoroscopy times. 


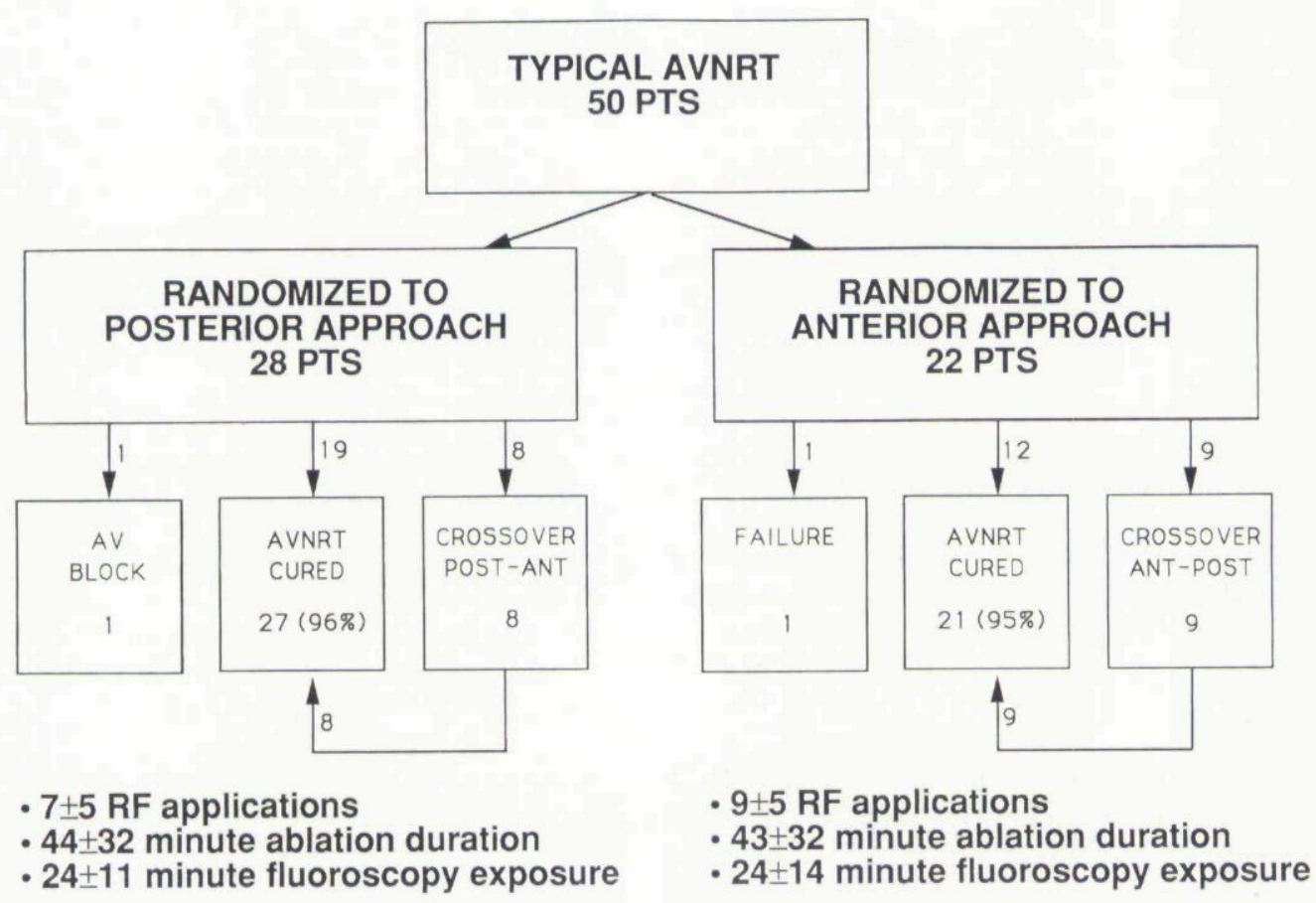

Figure 8. Overall outcome of patients randomized to receive lesions anteriorly versus posteriorly. Note that the eight patients who failed attempts at slow pathway ablation posteriorly were cured with anterior lesions with no inadvertent complete AV block.

portions of patients in both groups. Despite our relative inexperience, the posterior approach appeared no more difficult than the anterior as reflected by comparable numbers of radiofrequency energy applications, ablation session duration, and fluoroscopy exposure.

The overall outcome of the 50 patients in this study is summarized in Figure 8. All 17 patients who crossed over from one approach to the other were successfully cured. This included eight patients who continued to have inducible AV nodal reentry despite attempts at posterior ablation. All of these patients went on to be successfully cured of their AV nodal reentry with anterior lesions without inadvertent complete AV block.

\section{Summary and Clinical Implications}

Radiofrequency catheter ablation anteriorly appears to selectively affect fast AV nodal path- way function in patients with typical AV nodal reentry tachycardia. Although it is effective, it is associated with a significant risk of inadvertent complete AV block. Titration of radiofrequency power during the anterior approach reduces the risk of inadvertent complete AV block. This stepwise technique does not compromise efficacy and should be used whenever lesions are made in proximity to the distal AV junction.

The efficacy of the posterior approach is comparable to or greater than that of the anterior approach. It does not produce first degree AV block. By virtue of being a long distance from the distal AV node, the posterior approach dramatically reduces the risk of high grade AV block as well. For these reasons, the posterior approach should be the technique of first choice for radiofrequency ablation of typical AV nodal reentry. Occasionally, patients will fail the posterior approach. If AV nodal tachycardia remains inducible, it appears to be reasonable to cross over to the anterior approach. 


\section{References}

1. Lee MA, Morady F, Kadish A, et al. Catheter modification of the atrioventricular junction with radiofrequency energy for control of atrioventricular nodal reentry tachycardia. Circulation 1991; 83:827-835.

2. Ehrlich SS, Cannom DS, Dalen NA, et al. Radiofrequency catheter ablation of atrioventricular nodal reentry tachycardia by retrograde atrial mapping during tachycardia: A new ablation technique. (abstract) PACE 1992; 15:525.

3. Langberg JJ, Kim YN, Goyal R, et al. Conversion of typical to "atypical" atrioventricular nodal reentrant tachycardia after radiofrequency catheter modification of the AV junction. Am J Cardiol 1992; 69:503-508.

4. Jazayeri MR, Hempe SL, Sra JS, et al. Selective transcatheter ablation of the fast and slow pathways using radiofrequency energy in patients with atrioventricular nodal reentrant tachycardia. Circulation 1992; 85:1318-1328.
5. Langberg JJ, Calkins H, El-Atassi R, et al. Radiofrequency catheter ablation of the fast AV nodal pathway can be performed safely by titrating power output. (abstract) PACE 1992; 15:524.

6. Haissaguerre M, Gaita F, Fischer B, et al. Elimination of atrioventricular nodal reentrant tachycardia using discrete slow potentials to guide application of radiofrequency energy. Circulation 1992; 6: 2162-2175.

7. Kay GN, Epstein A, Dailey S, et al. Selective radiofrequency ablation of the slow pathway for the treatment of atrioventricular nodal reentrant tachycardia: Evidence for involvement of perinodal myocardium within the reentrant circuit. Circulation 1992; 5:1675-1688.

8. Leon A, El-Atassi R, Borganelli M, et al. A prospective, randomized comparison of anterior and posterior approaches for radiofrequency catheter modification of AV node reentry tachycardia. (abstract) J Am Coll Cardiol 1992; 19:145A. 
This document is a scanned copy of a printed document. No warranty is given about the accuracy of the copy. Users should refer to the original published version of the material. 